\title{
Size tunable nanoparticle formation employing droplet fusion by acoustic streaming applied to polyplexes
}

\author{
Lukas G. Schnitzler ${ }^{1}$, Stefanie Junger ${ }^{1}$, Dominik M. Loy ${ }^{2,3}$, Ernst Wagner ${ }^{2,3,4}$, Achim \\ Wixforth $^{1,3,4,5}$, Andreas Hörner ${ }^{1}$, Ulrich Lächelt ${ }^{2,3,4}$ and Christoph Westerhausen ${ }^{1,3,4,5^{*}}$ \\ 1 Chair for Experimental Physics 1, University of Augsburg, Universitätsstr. 1, 86159 Augsburg, Germany \\ 2 Pharmaceutical Biotechnology, Department of Pharmacy, Ludwig-Maximilians-Universität, 81377 Munich, \\ Germany \\ 3 Nanosystems Initiative Munich, Schellingstraße 4, 80799 Munich, Germany \\ 4 Center for NanoScience (CeNS), Ludwig-Maximilians-Universität Munich, 80799 Munich, Germany \\ 5 Augsburg Center for Innovative Technologies (ACIT), 86159 Augsburg, Germany
}

* Correspondence: christoph.westerhausen@gmail.com; Tel.: +49-821-598-3311

Abstract

Automated mixing of fluids with control over mixing parameters is of highest importance for reproducible production and chemical synthesis processes. We here introduce Surface Acoustic Waves (SAW) induced mixing of $\mu \mathrm{L}$ droplets for tailorable nanoparticle (NP) formation. Nucleic acid therapeutics represent extremely potent and innovative approaches to a variety of medical challenges, such as the treatment of cancer and genetic diseases. In this study, we apply this method to produce nucleic acid polymer complexes. Fusing two droplets containing either pDNA or cationic polymers leads to the formation of well-defined polyplexes. We show that droplet size and incubation time do not influence the desired particle characteristics significantly. However, the resulting nanoparticle diameter strongly depends on the SAW power level and educt concentrations, which indicates a kinetically controlled assembly process, while the particle shape is largely unaffected. Applying our novel technique to the formation of three-component-NP, we find that the choice of the mixing order can be used to decrease NP size even further. To address the kinetic interplay between mixing and particle growth, we apply our technique to homogenous mixing at high salt concentrations followed by a subsequent dilution step. Finally, by comparing various hand- and SAW-mixed polyplexes, we demonstrate significant differences in size while the cytotoxicity and in vitro efficacy remain roughly the same.

Keywords:

microfluidic mixing, droplets, surface acoustic waves, polyplexes, therapeutic nanoparticles 


\section{Introduction}

Nanopharmaceuticals make use of specific properties of nanosized objects in a biological environment for the controlled delivery of therapeutic agents. Since some types of tumors are extremely well accessible by nanoparticles due to a leaky vasculature, passive targeting of nanocarriers by the so called 'enhanced permeability and retention effect' (EPR) is an attractive approach for cancer treatments. Since common chemotherapeutic agents are accompanied with several side effects, delivering drugs in a protected and targeted manner is of utmost importance.

Several systems of therapeutic nanoparticles (NP) already exist [1]. All of those formulations have an improved side-effects profile by reducing the impact on healthy tissue. In this work, we focus on the formation process of such NP in a microfluidic approach. To be more precise, we focus on the formation of so called polyplexes [2] which are used as nucleic acid therapeutics [3]. Well-established and commonly used is a particle system containing plasmid DNA ( $p D N A$ ) and branched polyethylenimine (PEI). During mixing of these components, the particles are formed by ionic interactions between the negatively charged nucleic acid and the multiple positive charges of the polymer PEI $(25 \mathrm{kDa})$ at neutral $\mathrm{pH}$ [4-6]. Polyplex sizes and polydispersities were found to be strongly dependent on mixing speeds, with standardized use of micro-mixers being superior over classical pipetting [7]. More sophisticated polyplex systems commonly use three-component formulations of pDNA and sequence-defined cationic oligomers with polyethylene glycol shielding and folic acid or c-met binding pepide (CMBP) as receptor targeting ligands $[8,9]$.

NPs are usually produced in small volumes of solvent $(\mu \mathrm{L})$ and therefore microfluidic effects like the absence of turbulent mixing have to be considered. In the microfluidic regime, mixing is mostly based on diffusion and thereby slow. Several approaches exist to overcome this challenge [10-13]. Employing Surface Acoustic Waves (SAW) can be used to mix fluids by chaotic advection [14]. There already exist attempts to use SAW mixing to produce therapeutic nanoparticles in microchannels $[15,16]$. Droplet-based approaches offer various advantages over such high throughput methods for rapid testing and working with low volumes, without sacrificing the potential for scale up using parallelization and pipetting robots or other conventional dispensing units. The underlying physics of these SAW-based manipulation of small droplets were shown before. [17-19].

In this work, we leverage the potential of controlled droplet fusion and subsequent mixing by chaotic advection. The reduction of spatial and temporal inhomogeneities during the mixing process as they appear in common manual bulk mixing is shown. In conclusion, we can directly influence the mixing process to tailor the NP's size to exactly fit the intended application. 


\section{Experimental}

\section{SAW-Chip}

For the generation of SAW, which are employed to induce the mixing of $\mu \mathrm{L}$ droplets, we fabricated Inter-Digital-Transducers (IDT) of Ti-Au-Ti ( $5 \mathrm{~nm}-50 \mathrm{~nm}-5 \mathrm{~nm}$ height) on a $\mathrm{LiNbO}_{3}$ (128 ${ }^{\circ}$ rot $Y$-Cut) substrate. An IDT with 40 finger pairs with a periodicity $p=50 \mu \mathrm{m}$ between each finger and an aperture of $\mathrm{W}=600 \mu \mathrm{m}$ was used. To protect the multifinger electrodes, they are covered by a thin film of $\mathrm{SiO}_{2}(200 \mathrm{~nm})$. By incubating the substrate at low pressure (1 mbar) for $24 \mathrm{~h}$ with the chemical $1 \mathrm{H}, 1 \mathrm{H}, 2 \mathrm{H}, 2 \mathrm{H}$-Perfluordecytrichlorsilane (abcr $\mathrm{GmbH}$, Karlsruhe, Germany) a permanent hydrophobic surface is generated. Thereby the contact area between the droplet and the surface is smaller, which makes it easier to translocate them. The SAW frequency is $f=77.4 \mathrm{MHz}$ and typical values for the reflection $\mathrm{S}_{11}$ are around $-30 \mathrm{~dB}$, determined by a network analyzer (for details see supporting information). Typical voltages are $7 \mathrm{~V}$ (peak) according to $P=27 \mathrm{dBm}$. Signal Generators (CellEvator, Advalytix, München, Germany) with customized Lab-View-based control software and standard SMA-connectors were used.

\section{Mixing Experiments}

To verify the SAW induced mixing quality, we used light microscopy in combination with a high speed camera (FASTCAM 1024PCI, Photron, Ottobrunn, Germany). For the analysis process, one of the two fluids to be mixed was dyed with a food coloring (Patent Blue $V$ calcium salt, $1 \mathrm{mM}$, Sigma Aldrich, St. Louis, MO, USA). The videos were analyzed with the public domain software package ImageJ (1.48v, National Institutes of Health, Bethesda, MD, USA) [20]. The 8-bit grey scale videos yield discrete values ranging from 0 (black) to 255 (white).

\section{Droplet fusion by acoustic streaming}

The droplet fusion by acoustic streaming consists of two steps. In the first step, the droplet located closer to the IDT is pushed towards the second droplet by the SAW as reported earlier in detail [18] and one final droplet remains (fusion step). After fusion, the resulting droplet remains at this position and is now be mixed by internal acoustic streaming (mixing step). For the fusion step, we used different durations of the SAW pulses from $t_{\text {pulse }}=10 \mathrm{~ms}$ up to $t_{\text {pulse }}=$ $500 \mathrm{~ms}$. The subsequent mixing was performed using powers from $\mathrm{P}=5 \mathrm{dBm}$ up to $P=$ $27 \mathrm{dBm}$. The droplets in this proof of concept experiments were placed on the chip with a microliter-pipette (Research plus, Eppendorf, Hamburg, Germany). The first droplet was placed directly behind the IDT. The second droplet was placed behind the first one in a distance of a few mm. For the different experiments, droplet volumes between $\mathrm{V}=2 \mu \mathrm{L}$ and $\mathrm{V}=10 \mu \mathrm{L}$ were used.

\section{Polyplex formation}

The polyplex formation by acoustic streaming was performed as described above. The droplet closer to the IDT always contained plasmid DNA (pDNA, $10-160 \mu \mathrm{g} / \mathrm{mL})$ ) (pCMVLuc, Plasmid Factory, Bielefeld, Germany). The second droplet contained different types of polymer. Those are branched polyethylenimine (PEI, $12.5-200 \mu \mathrm{g} / \mathrm{mL}$ ) (PEI25, Sigma Aldrich, St. Louis, MO, USA), oligomers \#442, \#689 ,\#709 (synthesized as described earlier [8,9]), \#689:\#442:pDNA 2.3:8.2:1 (w/w/w) and \#689:\#709:pDNA 2.3:3.9:1 (w/w/w). In addition, we produced polyplexes by bulk mixing entitled 'hand mixing', where the two components were brought together in a microliter tube (PCR Tubes $0.2 \mathrm{~mL}$, Eppendorf, Hamburg, Germany) and mixed by pipetting up and down as fast as possible. 


\section{Particle image velocimetry experiments}

The SAW-generated flow field was characterized as described earlier [21]. In short, latex beads (Polysciences, Inc., Polybead $囚$, Polystyrene, diameter $3 \mu \mathrm{m}$ ) were added to the fluid in the droplet $(\mathrm{V}=10 \mu \mathrm{L})$. These particles are then used as tracers to follow the streamlines and make the fluid motion visible. For the analysis, the flow was recorded by a high-speed camera (Photron, FASTCAM 1024PCl). In a height of $\Delta z=200 \mu \mathrm{m}$ above the chip, 50 frames at a rate of $1000 \mathrm{fps}$ are captured. Large droplet deformations, however, as they occur at high SAW power levels of $P_{\text {SAW }}=26.5 \mathrm{dBm}$, make it difficult to apply the method above. Thus, an alternative approach was chosen here: spherical, fast streaming beads appear with an elongated shape in the recorded micrograph and from their lengths and the camera shutter time, the velocity was estimated.

\section{Three-component formulations and salt assisted formation}

The above described technique is used to produce so called three-component formulation NPs as well as polyplexes where the formation is salt assisted. The three-component formulation NP consist of pDNA and the polymers \#689 and \#709 or \#442. The formation was performed by subsequent mixing the components in different orders either by SAW mixing or by hand mixing. For the salt assisted formation, two droplets containing pDNA or PEI in $2 \mathrm{M} \mathrm{NaCl}$ are fused and mixed by SAW. Subsequently, the resulting droplet was fused and mixed with a larger droplet of deionized water, resulting in polyplex formation at a final isotonic concentration of $154 \mathrm{mM} \mathrm{NaCl}$.

\section{Particle size and PDI}

To characterize the NPs, Dynamic Light Scattering (DLS) was used. The final droplets were pipetted into DLS-cuvettes (PMMA, Brand, Wertheim, Germany). After $20 \mathrm{~min}$, the samples have been diluted with purified water and were then degassed for $10 \mathrm{~min}$ at approximately 175 mbar. For measuring the hydrodynamic diameter $d$ and the polydispersity index PDI the device 90Plus Particle Size Analyzer (Brookhaven Instruments Corporation, New York, NY, USA) was used. Parameters: temperature $20^{\circ} \mathrm{C}$, viscosity $1.002 \mathrm{cP}$, ref. index fluid 1.331 , angle $90^{\circ}$, wavelength $666 \mathrm{~nm}, 5$ Runs á 2 min, ref. index real 1.550 .

\section{Transmission electron microscopy}

Carbon coated copper grids (300 mesh, $3.0 \mathrm{~mm}$ O. D.) (Ted Pella, Inc. USA) were activated by plasma cleaning ( $420 \mathrm{~V}, 1 \mathrm{~min}$, argon atmosphere). Afterwards, five $\mu \mathrm{L}$ of the chosen polyplex solution ( 4 or $8 \mu \mathrm{g} / \mathrm{mL}$ pDNA, N/P $=6$, purified water) were incubated for three minutes on the grids before it was removed and stained by a $1.0 \%$ uranlyformate solution according to the following procedure: First, five $\mu \mathrm{L}$ uranylformate solution were placed on the grid and removed immediately, second, five $\mu \mathrm{L}$ of the same solution were left on the grid for five seconds before removal. Afterwards, the grids were dried for $30 \mathrm{~min}$ at room temperature. The stained polyplexes were visualized by a JEM/1011 transmission electron microscope.

\section{Gel-electrophoresis}

For the preparation of the gel, $1 \%[\mathrm{w} / \mathrm{v}]$ agarose was suspended in TBE buffer ( $89 \mathrm{mM}$ TRIS, $89 \mathrm{mM}$ borate, $2 \mathrm{mM}$ EDTA) and heated until the agarose was completely dissolved. Afterwards, $0.1 \%$ [v/v] GelRed (Biotium Inc., Hayward, CA, USA) was added and the solution was cast into an electrophoresis chamber. Samples containing $200 \mathrm{ng}$ pDNA were diluted to $20 \mu \mathrm{L}$ with purified water and supplemented with $4 \mu \mathrm{L}$ loading buffer ( $6 \mathrm{~mL}$ glycerol, $1.2 \mathrm{~mL}$ $0.5 \mathrm{M}$ EDTA solution $\mathrm{pH} 8.0,2.8 \mathrm{~mL}$ water, $20 \mathrm{mg}$ bromophenol blue) to a final volume of 24 $\mu \mathrm{L}$ and pipetted into the corresponding pockets of the solidified gel. As positive control a pDNA sample without polymer was used. The electrophoresis was run at $U=120 \mathrm{~V}$ for $80 \mathrm{~min}$. 


\section{Metabolic activity of transfected cells (MTT assay)}

Cells were seeded into 96 well plates $24 \mathrm{~h}$ prior to transfection. For the human prostate cancer cells DU145, each well contained 8.000 cells in $100 \mu \mathrm{L}$ RPMI-1640 medium with $10 \%$ FBS and $100 \mathrm{IU}$ penicillin and streptomycin each. For the HeLa derivative cells KB, each well contained 10000 cells in $100 \mu \mathrm{L}$ RPMI- 1640 folate free medium with the same amounts of FBS and pen/strep. Two hours before the cells were transfected, the medium in each well was replaced with $80 \mu \mathrm{L}$ fresh medium. Samples were prepared in HBG buffer (HEPES-buffered glucose, $20 \mathrm{mM} \mathrm{HEPES}$, pH 7.4, $5 \%$ glucose w/v) at room temperature and $100 \mu \mathrm{L}$ of each sample or control were distributed between five wells $(5 \times 20 \mu \mathrm{L})$. All plates have been incubated for $24 \mathrm{~h}$ at $37{ }^{\circ} \mathrm{C}$ in a humidified atmosphere with $5 \% \mathrm{CO}_{2}$. Afterwards, $10 \mu \mathrm{L} \mathrm{3-}$ (4,5-dimethylthiazol-2-yl)-2,5-diphenyltetrazoliumbromide (MTT) were added to each well and each plate was incubated for another two hours at $37^{\circ} \mathrm{C}, 5 \% \mathrm{CO}_{2}$. To release the purple dye from the cells, media was removed and the plates were frozen for at least one hour at $-80^{\circ} \mathrm{C}$. Subsequently, $100 \mu \mathrm{L}$ DMSO were filled in each well and incubated for $30 \mathrm{~min}$ at $37^{\circ} \mathrm{C}$ under gentle shaking. Finally, the absorbance of each well was measured at $590 \mathrm{~nm}$ against a 630 $\mathrm{nm}$ reference wavelength in a TECAN Spectra Fluor Plus plate reader (Tecan Trading AG, Switzerland) and the results were normalized to the mean of the wells containing HBG only.

\section{Luciferase assay}

The transfection process for this assay was identical to the process described in the MTT assay paragraph. After the $24 \mathrm{~h}$ incubation period was over, the old medium was removed and $100 \mu \mathrm{L}$ cell lysis buffer (25 mM Tris, pH 7.8, 2 mM EDTA, 2 mM DTT, $10 \%$ glycerol, $1 \%$ Triton $\mathrm{X}-100$ ) were added to each well and each plate was incubated for $45 \mathrm{~min}$ at room temperature. $35 \mu \mathrm{l}$ of each well were transferred to a opaque measurement plate and luciferase activity was measured in a Centro LB 960 plate reader luminometer (Berthold Technologies GmbH \& Co. $\mathrm{KG}$, Bad Wildbad, Germany) with LAR buffer (20 mM Glycylglycine, $1.0 \mathrm{mM} \mathrm{MgCl}_{2}, 0.1 \mathrm{mM}$ EDTA, $3.29 \mathrm{mM}$ DTT, $0.548 \mathrm{mM}$ ATP, $1.30 \mu \mathrm{M}$ coenzyme A, adjusted to $\mathrm{pH} 8.5$ with $\mathrm{NaOH}$ ) and luciferin (10 mM luciferin - Na, $29.375 \mathrm{mM}$ glycylglycine, adjusted to $\mathrm{pH} 8.5$ with $\mathrm{NaOH}$ ) at $21: 1$. Integration time per well was $10 \mathrm{~s}$. The mean of five wells per sample in relative light units (RLU) is reported. 


\section{Results and Discussion}

To evaluate the SAW induced droplet fusion, we first characterize the influencing factors of droplet volume and incubation time on the particle size. In the next steps we optimize the SAW induced mixing process. To do so, we quantify the mixing time depending on the SAW pulse duration during the initial droplet fusion and the subsequently applied mixing power as well as their influence on polyplex formation.

After careful characterization of our setup, it is applied to more modern three-component NP formations with respect to the influence of mixing order on size and polydispersity as well as mixing of two-component formulations at high salt concentrations to suppress premature electrostatic polyplex formation before SAW mixing is complete.

Finally, we study the in vitro efficacy of our polyplexes by using MTT assays to determine the metabolic activity and luciferase assays to measure the reporter gene expression of the transfected plasmid.

\section{Tunable nanoparticle size by variation of concentration and acoustic power}

The basic setup being used in all our experiments is illustrated in Figure 1. In front of the aperture of an interdigital transducer (IDT) two droplets are positioned. The droplet closer to the IDT contains pDNA, while the second droplet contains specific cationic polymers. By applying a radio frequency signal to the IDT, a travelling surface acoustic wave (SAW) is generated.

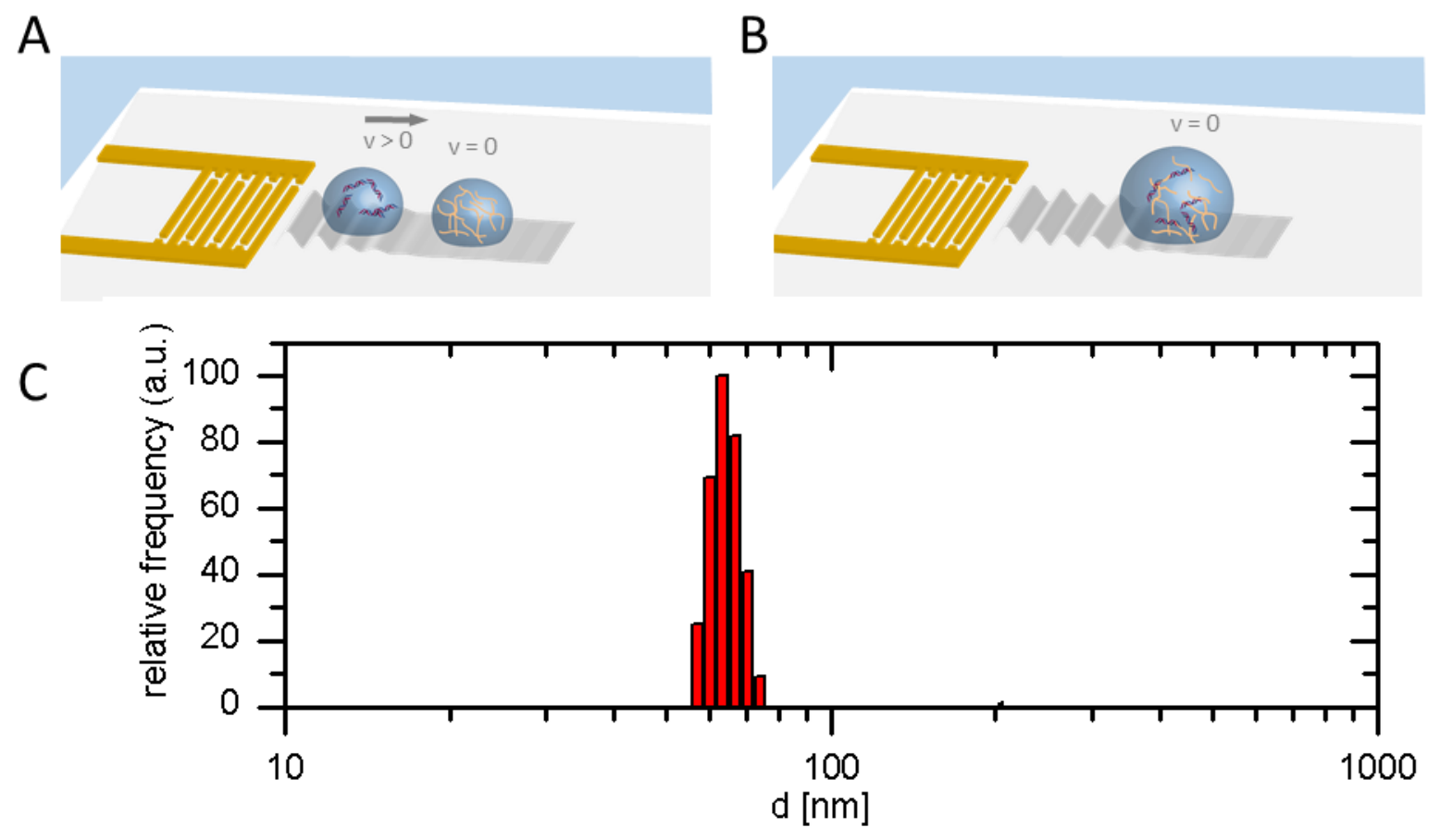

Figure 1: Experimental setup A) Two $\mu L$ droplets containing plasmid DNA and cationic polymers in aqueous solution are positioned in the sound path of a hydrophobic surface acoustic wave (SAW) chip. A short SAW pulse moves the PDNA containing droplet towards the polymer containing droplet $B$ ) In the fused droplet the oppositely charged components mix by diffusion or diffusion accelerated by 
SAW streaming and form ionic complexes C) Outcome of a typical experiment: Size distribution of NP produced by droplet fusion and mixing by SAWs (here PDNA + PEI) determined by DLS.

As soon as the SAW impinges on the first droplet, the sound wave is diffracted into the droplet and the amplitude of the so called leaky SAW on the substrate decays exponentially with a decay length $l$ of about $l \approx 10 \lambda_{S A W}$ (here $\lambda_{S A W}=50 \mu \mathrm{m}$ ) [22]. Thus, the second, polymer containing droplet experiences no significant acoustic force and stays unaffected. In the pDNA containing droplet in contrast, the sound wave leads to acoustic streaming and thus stirring within the droplet. As shown earlier by our group [23], employing a pulsed SAW with a sufficiently high SAW amplitude pushes the pDNA droplet towards the polymer droplet until they fuse. During fusion and subsequent stirring or diffusion, polymers form polyplexes with nucleic acids. Stirring is done with a reduced SAW amplitude. The polymers ( $m \sim 3 \mathrm{kDa})$ are rich in secondary amines that have a positive charge at neutral $\mathrm{pH}$, facilitating ionic interactions with the negatively charged nucleic acid. Figure $1 \mathrm{C}$ shows the NP size distribution of a typical experiment (here pDNA + PEI) determined by DLS. The damping of the SAW amplitude along the droplet-free sound path is negligible [24]. Therefore, the droplets can easily be placed manually with a microliter pipet without influencing the outcome. However, there are several parameters that can be adjusted to influence NP properties. Thus, in the following we investigate the influence of droplet volume, incubation time after particle formation, SAW amplitude and mixing time on the NPs' size in detail.

One of the easily adjustable parameters is droplet volume. Figure 2 shows NP diameter and polydispersity index (PDI) as a function of droplet volume. In a reasonable range (depending on the SAW chip) of droplet volumes $\boldsymbol{V}$ between $\mathbf{1} \boldsymbol{\mu l} \leq \boldsymbol{V} \leq \mathbf{1 0} \boldsymbol{\mu l}$, no significant changes of both mean NP diameter and mean PDI is observed. This provides flexibility and indicates robustness and reliability of the SAW-assisted mixing process. Working with smaller volumes is still possible, but more difficult to handle. To avoid significant evaporation during the experimental time, either steam saturated atmosphere or oil coatings of the droplets can be used [25]. For pragmatic reasons, however, we do not follow this approach in this study.

A second important parameter to check regarding its impact on the NP size is the incubation time after polyplex formation. Particle diameters determined by DLS immediately, three and seven days after formation exhibit a stable particle size and only a very slight compacting of the NP over the time of seven days (2.4\% for 72 hours $-2.9 \%$ for 144 hours). However, this effect is still in the order of error bars (data not shown).

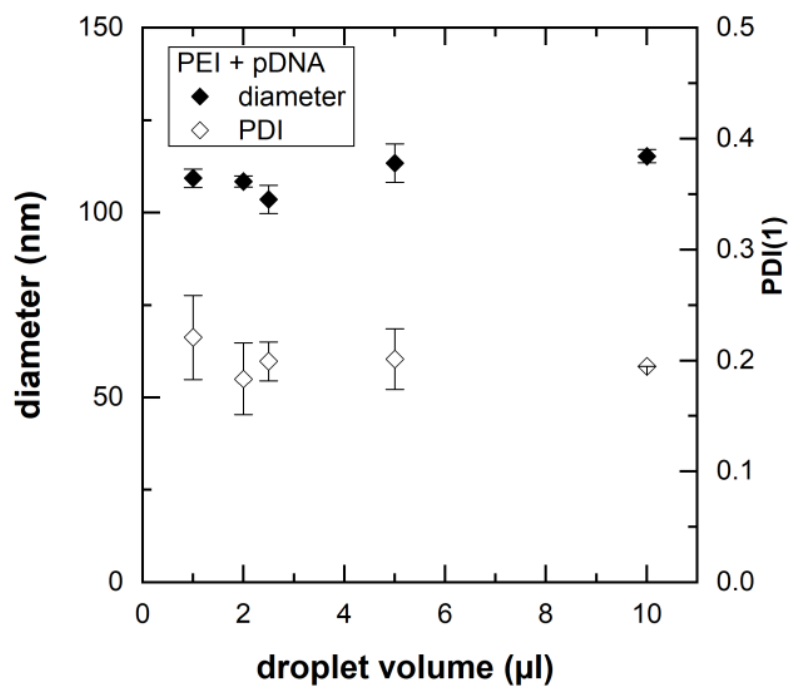

Figure 2: NP size as function of droplet volume NP diameter and PDI as function of droplet volume (PEI $\left.+p D N A, c_{P E I}=104 \mu \mathrm{g} / \mathrm{mL}, c_{P D N A}=80 \mu \mathrm{g} / \mathrm{mL}, P_{\text {pulse }}=26.5 \mathrm{dBm}, t_{\text {pulse }}=50 \mathrm{~ms}, t_{\text {mixing }}=750 \mathrm{~ms}\right)$. 
Next, we studied pulse duration, SAW mixing power and time. First, we quantitatively measure a previously introduced dimensionless mixing parameter [16] as function of the duration of the applied SAW pulse for the fusion process and SAW amplitude for the subsequent mixing step. The mentioned mixing parameter $\widetilde{M}$ is given by the mean grey scale value of the analyzed region normalized to the standard deviation:

$$
\widetilde{M}=\frac{\bar{X}}{\sigma}=\frac{\bar{X}}{\sqrt{\frac{1}{n-1} \sum_{i=1}^{n}\left(X_{i}-X\right)^{2}}} .
$$

Here, $\bar{X}$ is the arithmetic mean grey value, $\sigma$ is the standard deviation, $n$ is the number of pixels and $X_{i}$ is the grey scale value at the position $i$. A homogenous distribution of the grey scale values results in small values of $\sigma$, which in turn leads to large values of $\sigma^{-1}$. To compensate the influence of slightly different illumination for the different micrographs and to ensure comparability, $\sigma^{-1}$ is scaled with $\bar{X}$. To ensure comparability and intuitive understanding of the results, we normalize the difference of the mixing parameter $\widetilde{M}$ for SAW-mixing and diffusive mixing (SAW off) to the interval $[0,1]$ and entitle it as 'mixing efficiency':

$$
M=\frac{\widetilde{M}-\widetilde{M}_{\min }}{\widetilde{M}_{\max }-\widetilde{M}_{\min }}
$$

where $\widetilde{M}_{\min }$ is the value of the unmixed and $\widetilde{M}_{M a x}$ the value of the mixed state.

Figure $3 \mathrm{~A}$ shows micrographs of corresponding experiments using a coloured solution as described in detail in the materials \& methods section. In the central region of the droplet, where the influence of the droplet edges is minimal, we determine $M$ as function of time. Directly after fusion ( $\mathrm{t}=3 \mathrm{~ms}$ ) the final droplet is not completely mixed. Induced by further SAW mixing a homogenous mixture is obtained after $1 \mathrm{~s}$. Figure $3 \mathrm{~B}$ shows such $M(t)$ for various pulse durations without subsequent SAW mixing, while the SAW power $P_{\text {pulse }}$ is kept constant at $P_{\text {pulse }}=26.5 \mathrm{dBm}$. For very short pulses of $t_{\text {pulse }}=10 \mathrm{~ms}$ and without further stirring, droplet fusion and only partial mixing appears on the observed timescales. With increasing pulse duration, the distribution in the resulting droplet becomes more and more homogeneous. To study the impact of the mixing power, the shortest possible pulse duration of our setup $t_{\text {pulse }}=$ $10 \mathrm{~ms}$ was chosen with subsequent mixing for $10 \mathrm{~s}$ at different SAW powers $P$ between $5 \mathrm{dBm}$ and $26.5 \mathrm{dBm}$. The corresponding time dependent mixing parameters during these experiments are shown in Figure $3 \mathrm{C}$. With increasing $P$, the time until a homogeneous distribution is reached decreases from $10 \mathrm{~s}$ for $P=5 \mathrm{dBm}$ to $1 \mathrm{~s}$ for $P=26.5 \mathrm{dBm}$. Without SAWs, only very slow diffusive mixing is observed. 
A
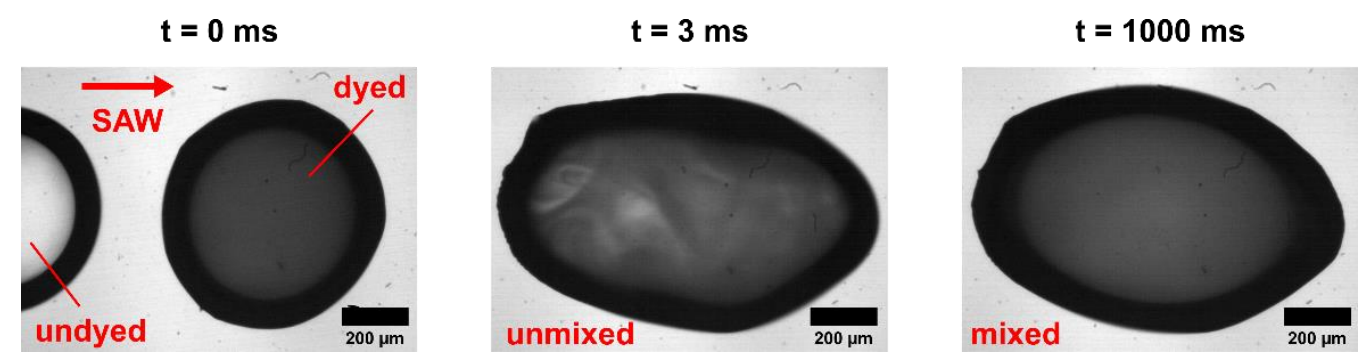

B

C
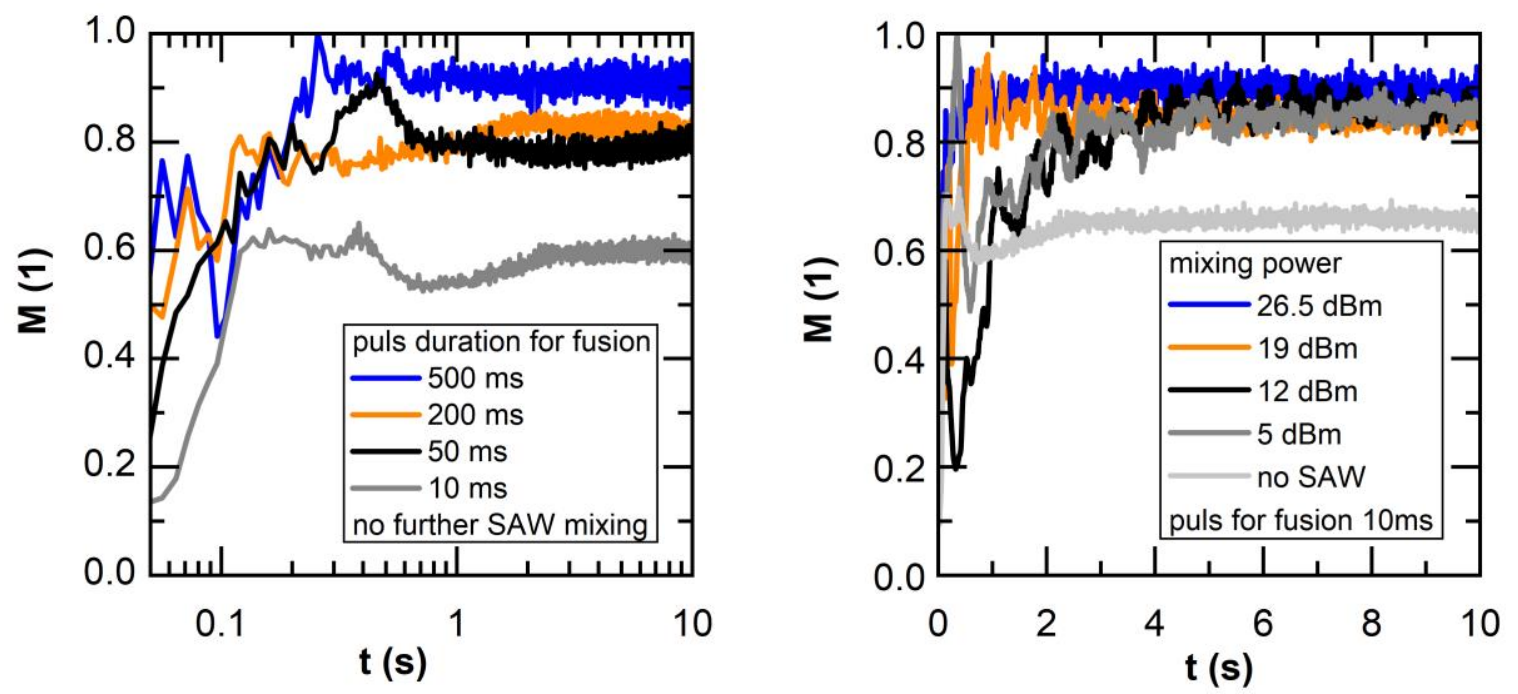

Figure 3: Mixing quality as function of SAW induced fusion and mixing A) Exemplary micrographs of time sequences of mixing processes of two $2 \mu \mathrm{L}$ droplets prior to and after SAW induced fusion. One droplet is dyed using a $1 \mathrm{mM}$ patent blue solution, while the second droplet consists of pure water. B) Mixing quality according to eq. 2 as function of time for different SAW pulse durations during the fusion process without further acoustic mixing. C) Mixing quality after droplet fusion by a 10 ms pulse and subsequent SAW mixing at different power levels $P$.

In analogy to the fusion and mixing experiments shown in Figure 3, NPs consisting of PEI and pDNA are produced by fusion of two droplets $(V=10 \mu \mathrm{L})$. Figure 4A shows the mean NP diameter for different pulse durations between $10 \mathrm{~ms}$ and $500 \mathrm{~ms}$ without subsequent stirring. Particles show a mean diameter $d=216 \mathrm{~nm}$ independent of the pulse duration. While the diameter does not change significantly, its standard deviation slightly increases with increasing pulse duration. Obviously, the NP's diameter does not exeedingly depend on $t_{\text {pulse }}$. In contrast, the SAW power during mixing by acoustic streaming after droplet fusion turns out to be a key parameter. Figure 4B shows the mean NP diameter of similar experiments with SAW induced chaotic advection mixing at different powers $P$ in the interval $5 \mathrm{dBm} \leq P \leq 26.5 \mathrm{dBm}$. Already gentle stirring at $P=5 \mathrm{dBm}$ results in a significant smaller NP diameter $d=169 \pm 17 \mathrm{~nm}$ and clearly decreases further with increasing SAW power. The highest applied power $P=$ $26.5 \mathrm{dBm}$ results in NP of diameters $d=87 \pm 2 \mathrm{~nm}$. This dependence of the NP diameter on $P$ is discussed below together with the impact of educt concentrations. This decrease in size with increasing SAW power goes hand in hand with an increase in streaming velocity (Figure 4B). 

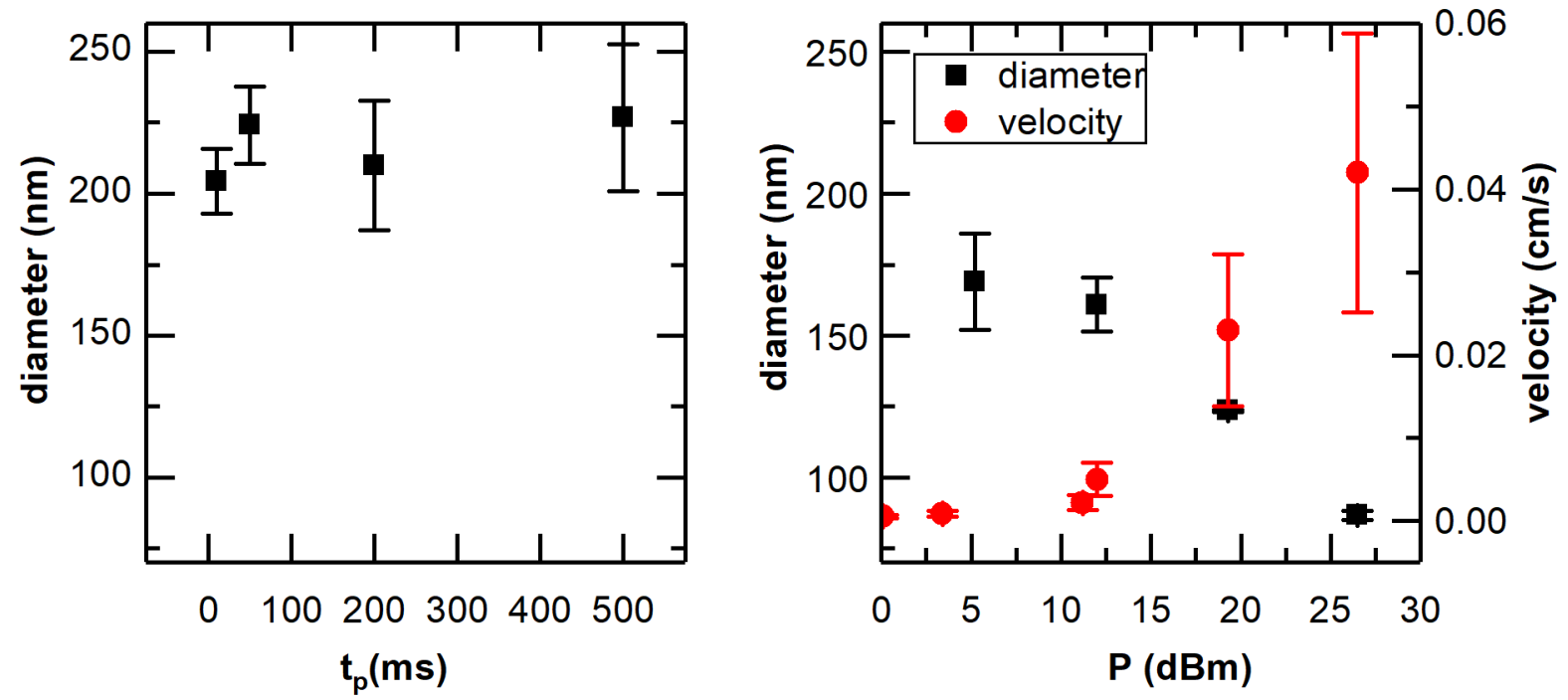

Figure 4: Mean NP diameter as function of SAW induced droplet fusion and mixing

A) Mean NP diameter as function of the length of the droplet fusion pulse without further stirring (10 $\mu L P E I+10 \mu L p D N A, c_{P E I}=104 \mu \mathrm{g} / \mathrm{mL}, c_{P D N A}=80 \mu \mathrm{g} / \mathrm{mL}, P_{\text {pulse }}=26.5 \mathrm{dBm}$ ) B) Mean NP diameter (black squares) as a result of droplet fusion (same concentrations and power as in $A, t_{\text {pulse }}=10 \mathrm{~ms}$ ) with subsequent mixing for $10 \mathrm{~s}$ using different SAW power $P$ and the maximal fluid velocity within the droplet in a height of $200 \mu \mathrm{m}$ above the chip surface (red circles).

While the NP size is strongly affected by the applied SAW power, the shape is not, as can be seen in Figure 5 and in the SI in more detail. Here, PEI and pDNA were hand or SAW mixed. As the overview micrographs in Figure $5 \mathrm{~A}$ and $5 \mathrm{C}$ show, hand mixing results in a wider size distribution and larger particles. The size but not shape control is in line with previous reports on thermodynamically stable finale states [26,27]. However, deviating from the results shown in Figure 4, we generally produce smaller particles, both for hand- and SAW mixing. The only deviation of adjustable parameters from the experiments with PEI shown above is the use of reduced $\mathrm{PEI}$ and pDNA concentration while the ratio PEI:pDNA is kept constant. This raises the question about the impact of concentration and if it influences polyplexes produced either by SAWs or hand mixing to a different degree. 

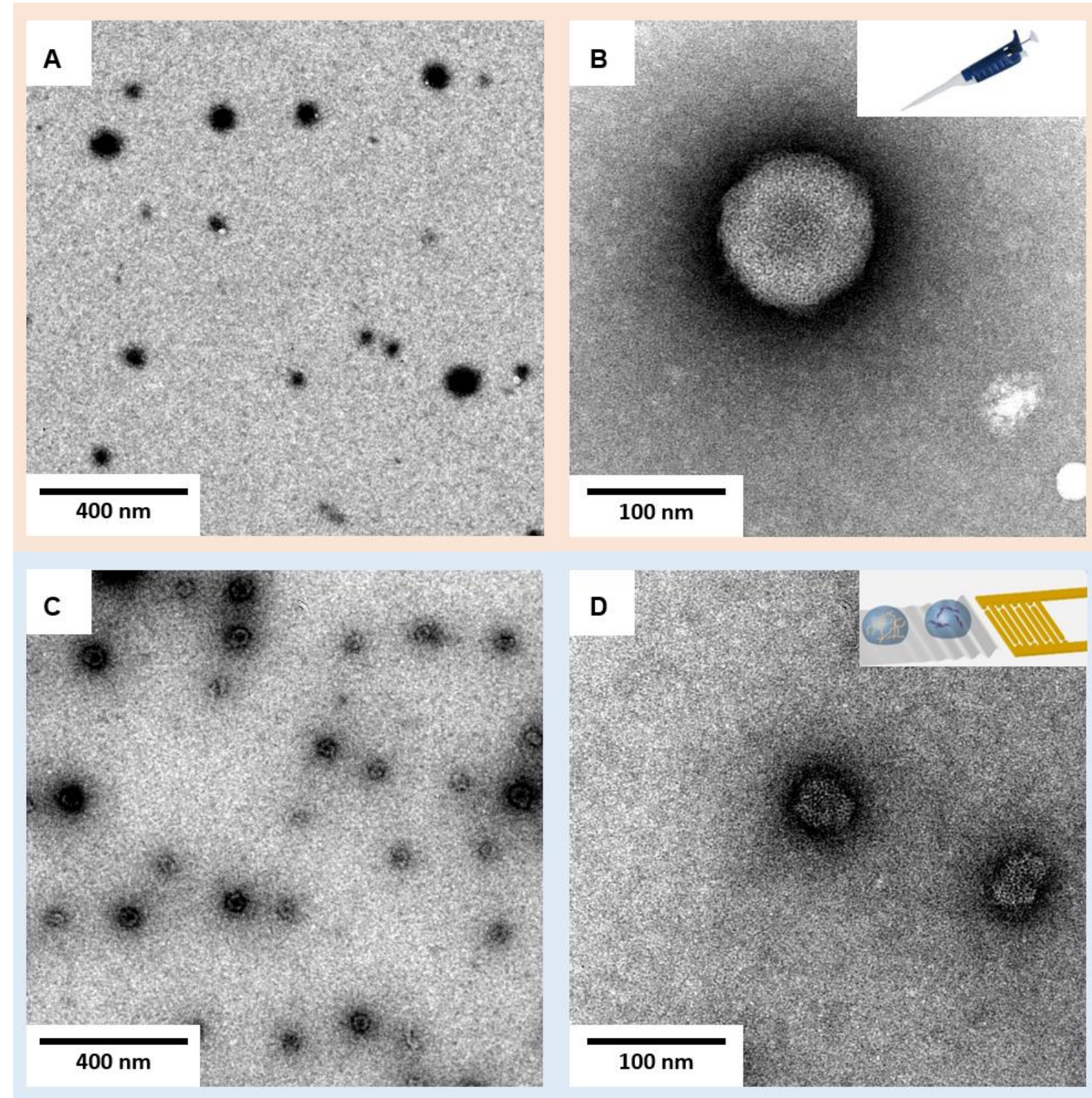

Figure 5: Comparison of NP morphology TEM images of hand mixed $(A, B)$ and SAW mixed polyplexes (C, D). $\left(10 \mu \mathrm{L} P E I+10 \mu \mathrm{L} D N A, c_{P E I}=52 \mu \mathrm{g} / \mathrm{mL}, c_{P D N A}=40 \mu \mathrm{g} / \mathrm{mL}, P_{\text {pulse }}=26.5 \mathrm{dBm}, t_{\text {pulse }}=10 \mathrm{~ms}\right)$

To address this question, polyplexes were produced employing both hand and SAW mixing $(P=26.5 \mathrm{dBm})$ for various concentrations of $\mathrm{PEI}$ and pDNA. Figure 6 shows the corresponding diameters determined by DLS. Within the studied concentration range, $d\left(c_{p D N A}\right)$ nicely fits to a linear function approximated by $d\left(c_{p D N A}\right) \approx 41 \mathrm{~nm}+1.6 \mathrm{~nm} \mathrm{ml} / \mu \mathrm{g}$. At very high concentrations, slight but systematic deviations appear. We attribute these deviations to a saturation of the SAW accelerated diffusion at high concentrations and thus decreasing mean intermolecular distances, where a linear relation is no longer valid. The range at which the size of polyplexes can be reliably tuned is wider for NPs produced with SAWs then with hand mixing. Increasing particle size with increasing concentration for a fix N:P ratio was reported earlier and is in line with our results [28]. Potential deviations between NP produced by different users (not studied explicitly here) are almost negligible using SAW induced droplet fusion and mixing. 


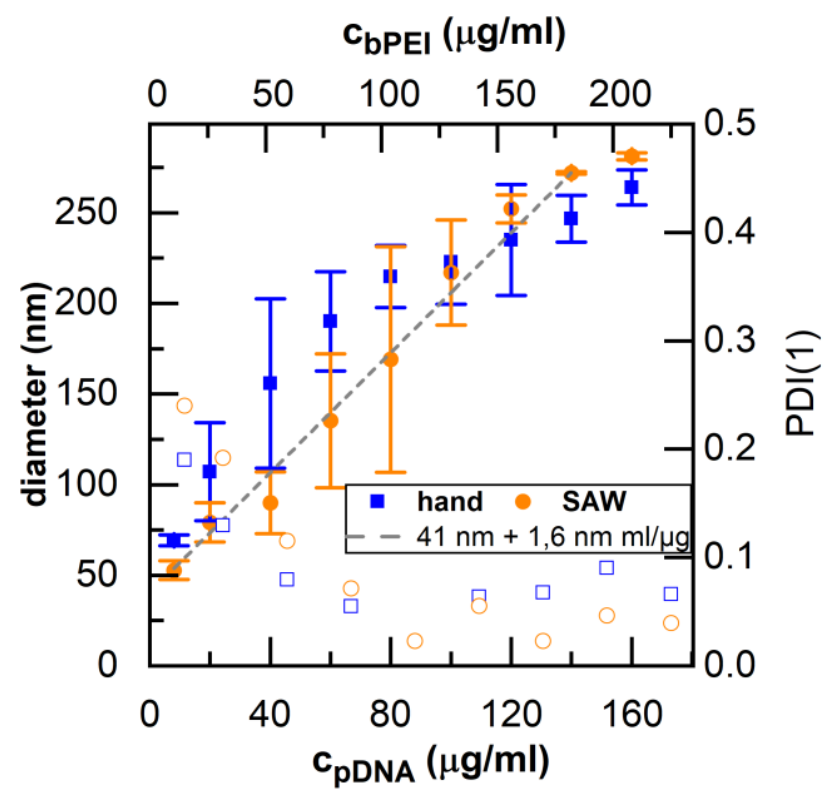

Figure 6: Concentration dependent mean NP diameter

Diameter of NP from manually or SAW mixed PEI and PDNA polyplexes, determined by DLS (10 $\mu \mathrm{L} P E I$ $\left.+10 \mu \mathrm{L} p D N A, c_{A P E} / c_{P D N A}=1.3, P_{\text {pulse }}=26.5 \mathrm{dBm}, t_{\text {pulse }}=10 \mathrm{~ms}\right)$. Data points and error bars indicate mean values and standard deviation of three independent experiments.

Summarizing the evaluation of main parameters, we found that droplet volume, incubation time after particle formation and duration of the SAW pulse for droplet fusion play a minor role, while SAW amplitude and educt concentration are the key parameters. For a constant concentration of $80 \mu \mathrm{g} / \mathrm{mL}$ mean NP size can be adjusted between $216 \mathrm{~nm}$ and $87 \mathrm{~nm}$ by tuning the SAW power $P$. Additionally, the particle diameter can be tuned nicely by changing the concentration without changing the ratio between educts. In the investigated concentration range, size and concentration are positively correlated. 


\section{Applications}

In this section, we apply the technique to the formation of multi-component NPs, electrostatic shielding at high salt concentration with subsequent dilution and particle formulation. Finally, we demonstrate significant differences in size for various SAW-mixed polyplexes while the cytotoxicity and in vitro efficacy are conserved.

\section{II.A Multi-component NP formulation}

Nanopharmaceuticals and gene carriers in general have to combine different functionalities to fulfill different tasks during the delivery route. To investigate the applicability of SAW-assisted droplet mixing to more sophisticated polyplex systems, a sequence-defined cationic three-arm core oligomer \#689 [8] and a sequence-defined cationic shell oligomer with polyethylene glycol segment and receptor targeting ligand folic acid (\#709 [9]) or c-met binding peptide (\#442 [8]) were used for the generation of three-component polyplexes with pDNA (Figure 7A). The combination of \#689 and \#442 has been shown to mediate efficient and receptor-specific transgene expression in vitro and in vivo. The presence of core oligomer \#689 had critical impact on pDNA condensation and tumor accumulation. Since the exact mixing protocol can play an important role, all possible orders for sequential mixing of the components were investigated by the SAW-induced droplet fusion method.

We produced polyplexes from two different polymers (Figure 7A) and pDNA. In the specific case we used the polymer A \#689, and the polymer B \#709, and mixed them with pDNA (C), (parameters: for each component $V=2 \mu L, P=26.5 \mathrm{dBm}$ ) by pushing a droplet of $A$ into a droplet of $C$ and likewise for $B$ and $C$. Figure 7B shows the mean diameter of the resulting particles. As expected from the branched structure of $B$, the $B C$ particles are somewhat larger than the AC particles. In the next step, we combined both polymers with pDNA in one NP. To do so, there are several options for pairwise mixing. The nomenclature $A B C$, used in the following paragraph, means a droplet of $A(V=2 \mu L, P=26.5 \mathrm{dBm})$ is fused and mixed with a droplet of $B$, the resulting droplet $(V=4 \mu L, P=26.5 \mathrm{dBm})$ is fused and mixed with a $2 \mu \mathrm{L}$ droplet of $C$. Reordering of the letters means a change of the fusion and mixing order. As a reference the analog procedure is applied to the solutions by hand mixing in a vial. 
A

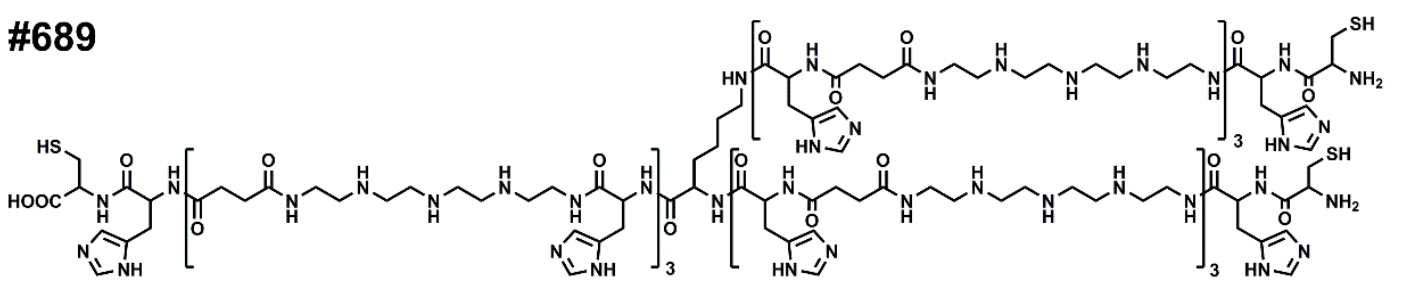

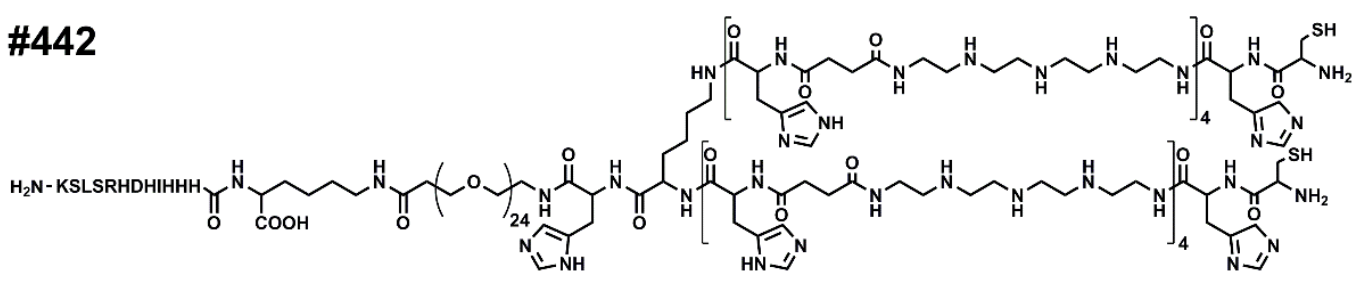

\#709

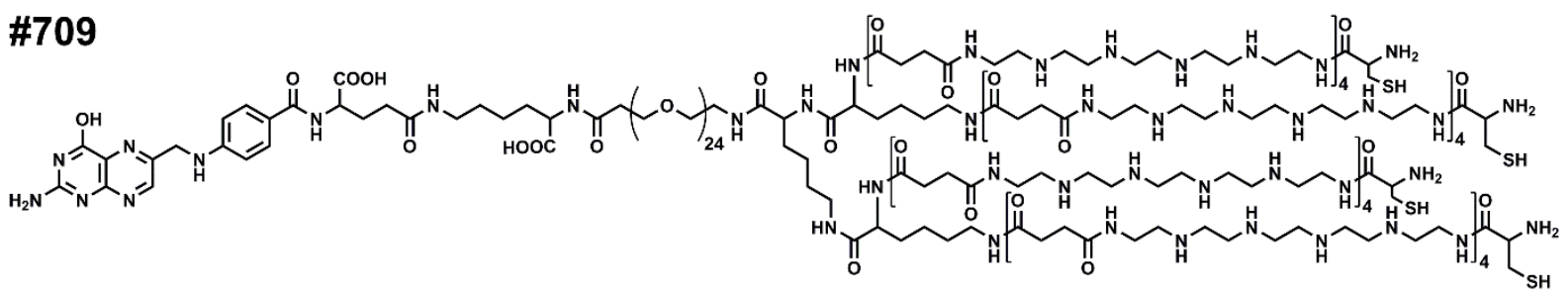

B

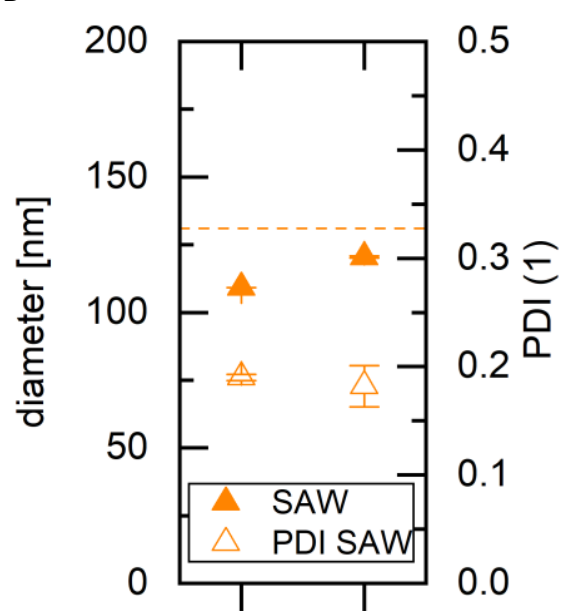

AC $\quad B C$

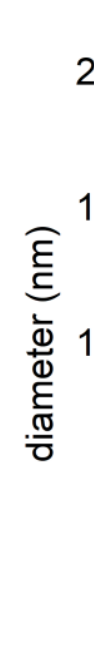

C 
mixing orders $A C B, B C A, C A B$ and $C B A$ where the pDNA is mixed with one of the two polymer components in the first step result in hydrodynamic diameters of $d=130 \pm 5 \mathrm{~nm}$. The mixing process $A B C$, where first both polymers $(A B)$ are mixed homogenously and then added to the pDNA (C) leads to compacter particles with a diameter of $d=104 \pm 2 \mathrm{~nm}$. One explanation could be that only in the order $A B C$ the electrostatic complexes are directly formed at optimal polymer to pDNA ratio which impacts DNA condensation[29,30]. However, the PDI in all experiments is not significantly different. Thus, the application of SAW mixing opens new opportunities to fine-tune NP's size.

\section{II.B Employing electrostatic shielding at high salt concentration}

The self-assembly of polyplexes is driven by electrostatic interactions and the increase of total entropy upon release of bound counter ions [31,32]. The electrostatic interactions strongly depend on the Debye length which is a function of ion concentration of the solvent. DeRouchey et al. showed structural transitions for various DNA-polycation complexes as function of the $\mathrm{NaCl}$ concentration of the solvent [33]. Following these authors, polyplexes exist in tight bundles, loose bundles and a network phase followed by dilute suspensions with increasing $\mathrm{NaCl}$ concentration. For PEl they find transition concentrations at about $500 \mathrm{mM}$ and $1.7 \mathrm{M}$, respectively. To obtain a homogeneous and uniform particle size distribution combined with physiological salt concentration to stabilize particles [34], we used the above introduced droplet based polymer and DNA mixing step at very high salt concentrations $(2 \mathrm{M} \mathrm{NaCl})$ and combined it with a droplet based dilution step. While the opposite charges of polymer (here \#709) and pDNA are largely screened during the mixing process, the situation changes drastically during the subsequent dilution step and polyplexes form at a final concentration of $154 \mathrm{mM} \mathrm{NaCl}$ as illustrated in Figure 8A.

This procedure leads to substantially larger particles with an average diameter $d_{H}=239 \pm$ $18 \mathrm{~nm}$ in presence of $\mathrm{NaCl}$ compared to a $\mathrm{NaCl}$ free environment with $d_{H}=90 \pm 4 \mathrm{~nm}$, as shown in Figure 8B. One potential reason could be a partial NP aggregation facilitated by the remaining $\mathrm{NaCl}$. However, the NPs produced in presence of salt show a decreased PDI of $P D I=0.102 \pm 0.014$ compared to the salt free experiment $(P D I=0.189 \pm 0.004)$. The same effect is observed with PEI / pDNA polyplexes. Within a concentration range of $25 \frac{\mu g}{m l} \leq c_{p D N A} \leq 75 \frac{\mu g}{m l}$, the concentration of pDNA and PEI have a strong impact on the NP diameter. For a constant ratio of $\frac{C_{b P E I}}{C_{P D N A}}=1.3$, the NP diameter increases linearly with increasing concentration, both in presence and absence of $\mathrm{NaCl}$, as shown in Figure $8 \mathrm{C}$. The equations describing this behavior best are (please note: fitting equations only showing the trend due to low number of data points):

$$
\begin{array}{ll}
\text { without } \mathrm{NaCl} & d_{H}=41 \mathrm{~nm} \pm 1.2 \frac{\mathrm{nm}}{\mu \mathrm{g} / \mathrm{ml}} \\
\text { with } \mathrm{NaCl} & d_{H}=71 \mathrm{~nm} \pm 2.4 \frac{\mathrm{nm}}{\mu \mathrm{g} / \mathrm{ml}}
\end{array}
$$

The PDI of the particles produced in presence of $\mathrm{NaCl}$ is significantly reduced in the lower concentration range, while the differences decrease with increasing concentration. In combination with the results presented above, this allows us to nicely tune the NP's diameter either by variation of the SAW power $P$, the use of $\mathrm{NaCl}$ or variation of the concentration. 


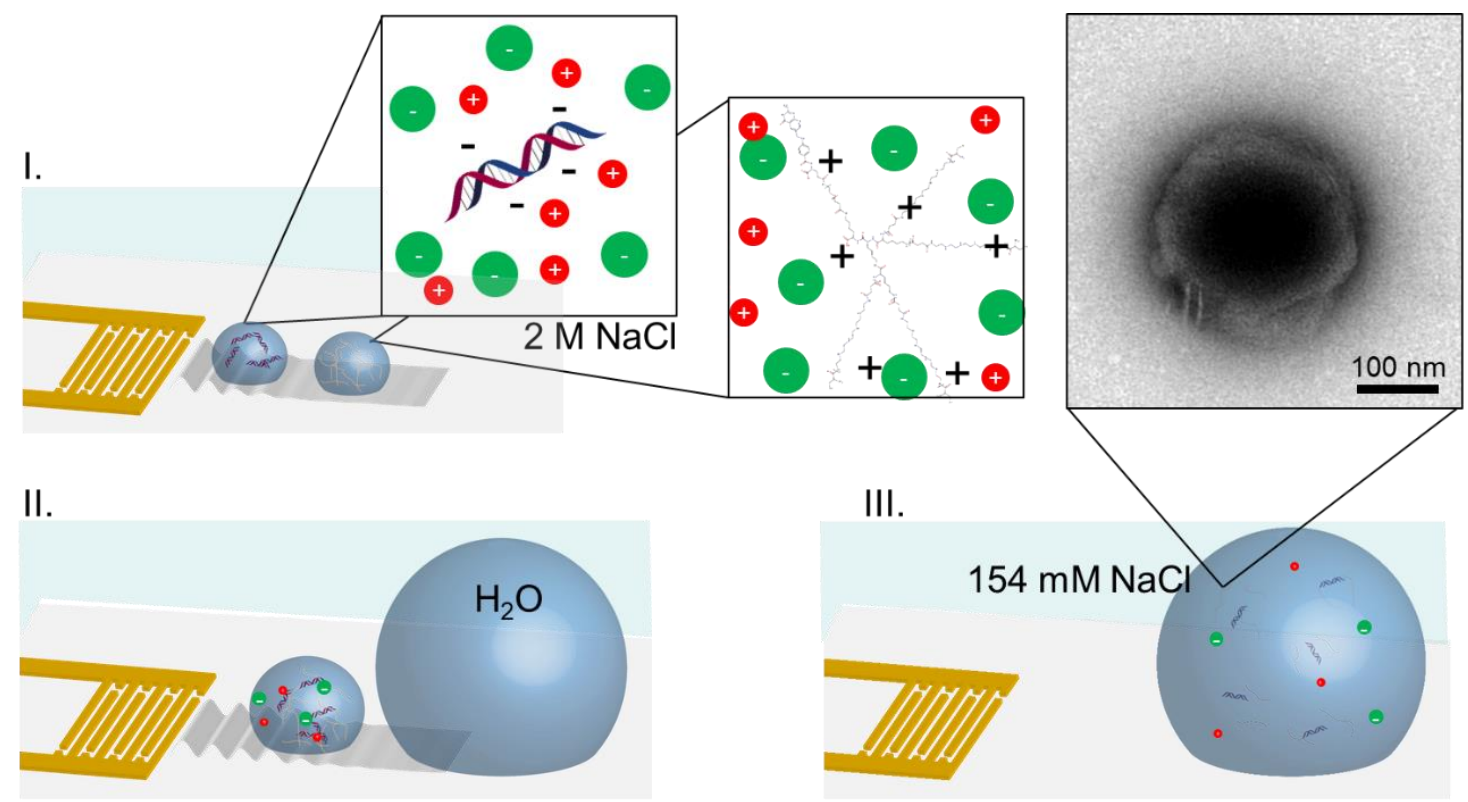

B

C

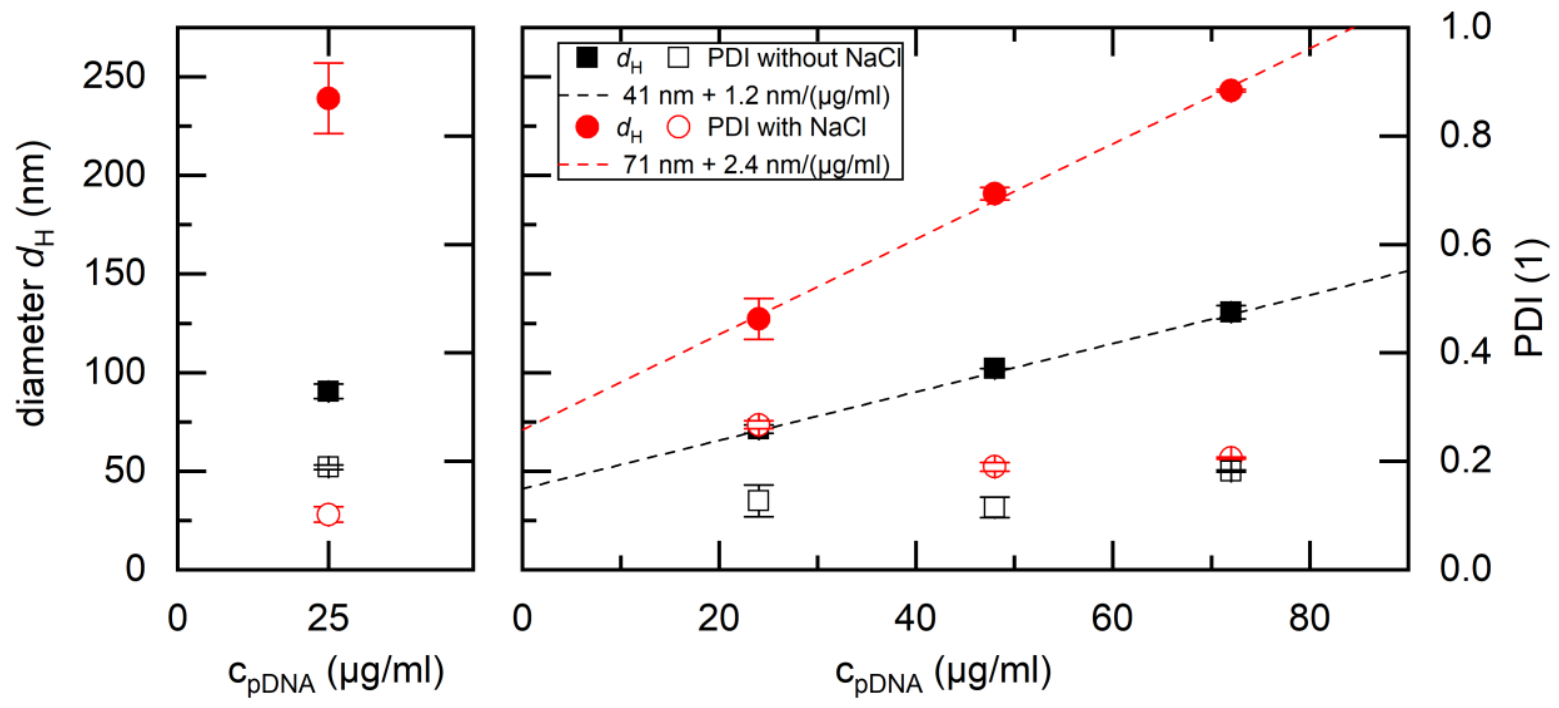

Figure 8: High salt concentration experiments A) Illustration of the experiment: I. two droplets containing PDNA and polymer \#709, each in $2 \mathrm{M} \mathrm{NaCl}$, are fused and mixed by SAW. A short Debye length prohibits polyplex formation. II. Subsequently, the resulting droplet is fused and mixed with a larger water droplet, III. resulting in polyplex formation at a final concentration of $154 \mathrm{mM} \mathrm{NaCl}$, as shown by the TEM micrograph in the inset. B) \#709 and pDNA: Diameter and polydispersity index PDI of such polyplexes from \#709 and pDNA produced by SAW induced droplet fusion and mixing in absence and presence of NaCl. $\left(2 \mu \mathrm{L}\right.$ polymer \#709 $+2 \mu \mathrm{L} p D N A$, each in $2 \mathrm{M} \mathrm{NaCl}, c_{\# 709}=258 \mu \mathrm{g} / \mathrm{mL}, c_{D D N A}=47$ $\mu \mathrm{g} / \mathrm{mL},+48 \mu \mathrm{L}$ water, $\left.P_{\text {pulse }}=26.5 \mathrm{dBm}, t_{\text {pulse }}=10 \mathrm{~ms}\right)$. C) PEI and pDNA: Diameter and polydispersity index $P D I$ of polyplexes as function of PDNA/PEI concentration produced by SAW induced droplet fusion and mixing in absence and presence of $\mathrm{NaCl}$. $\left(2 \mu L \mathrm{PEI}+2 \mu L \mathrm{PDNA}\right.$, each in $2 \mathrm{M} \mathrm{NaCl}, c_{A P E I} / c_{P D N A}=1.3$, $+48 \mu \mathrm{L}$ water, $P_{\text {pulse }}=26.5 \mathrm{dBm}, t_{\text {pulse }}=10 \mathrm{~ms}$ ).

Picking up the idea on SAW mixing interfering with the reaction kinetics can explain the results shown here. At low salt concentrations, mixing and particle formation cannot be distinguished clearly. High salt concentrations decrease or even prevent the electrostatic interaction of polymers and pDNA allowing almost perfect mixing of all components before particles are formed. The final dilution step regulates the NP size, since then the electrostatic interaction and complex formation are initiated. Similar to the interplay between mixing speed and particle 
growth observed under salt-free conditions, the dilution speed influences the polyplex size and larger particles are formed at slower dilution rates.

Finally, in the following section we address the question whether the SAW-assisted polyplex formation changes the in vitro efficacy for various polymer and nucleic acid combinations.

\section{II.C Conserved in vitro efficacy}

To study the in vitro applicability of SAW-mixed polyplexes, we assess their biological activity. To treat c-Met expressing DU145 cells (prostate cancer cells) and folic acid receptor expressing KB cells (cervical cancer cells) we prepared SAW and bulk mixed polyplexes from polymers \#442 (two drop system) and \#442 \& \#689 (three drop system) or \#709 and \#709 \& \#689 respectively. Based on the results shown in Figure 7, we first mixed the two polymers and subsequently fused the resulting droplet with the pDNA droplet in all three-droplet systems.

Figure 9A shows the gel ectrophoresis result of all formed polyplexes and free pDNA as a reference for pDNA binding capability. In all experiments, three-component NPs were successfully formed and no free pDNA was detected for both hand and SAW mixed NPs. In line with the results shown above, SAW-mixed NPs exhibit smaller diameters compared to hand mixed NPs while keeping all other variables constant: This can be deduced from Figure 9B. Moreover, polyplexes containing \#689 together with another polymer form smaller particles and exhibit a reduced PDI for SAW-mixed NPs. The same trend is visible for hand-mixed particles including polymers \#442 and \#689 but not \#709.
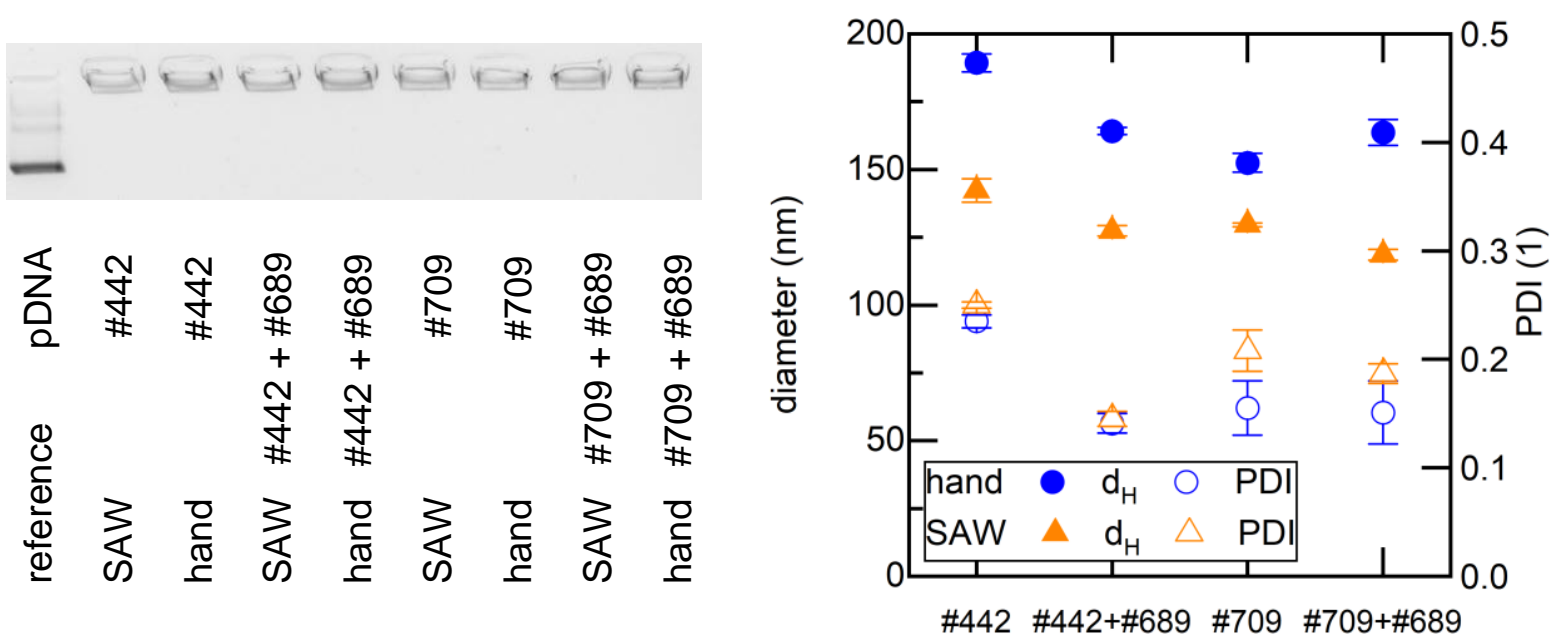

Figure 9: Polyplex formation and size A) Gel electrophoresis result of free pDNA and polyplexes from polymer \#442, \#709, \#689 and pDNA, hand mixed and SAW mixed. (Two component polyplexes: $2 \mu L$ polymer $+2 \mu L \mathrm{pDNA}, c_{\# 442}=1100 \mu \mathrm{g} / \mathrm{mL}, c_{\# 709}=517 \mu \mathrm{g} / \mathrm{mL}, c_{p D N A}=94 \mu \mathrm{g} / \mathrm{mL}, P_{\text {pulse }}=26.5 \mathrm{dBm}, t_{\text {pulse }}$ $=10 \mathrm{~ms}$. Three component polyplexes: $2 \mu \mathrm{L}$ polymer $A+2 \mu \mathrm{L}$ polymer $B+2 \mu L \mathrm{pDNA}, c_{\# 442}=782$ $\left.\mu \mathrm{g} / \mathrm{mL}, c_{\# 689}=213 \mu \mathrm{g} / \mathrm{mL}, c_{\# 709}=357 \mu \mathrm{g} / \mathrm{mL}, c_{p D N A}=94 \mu \mathrm{g} / \mathrm{mL}, P_{\text {pulse }}=26.5 \mathrm{dBm}, t_{\text {pulse }}=10 \mathrm{~ms}\right)$. B) Diameter and polydispersity index PDI of polyplexes from polymers \#442, \#709, \#689. The molecular structures of the polymers are shown in detail in the SI.

To quantify the transfection process and the cytotoxicity of the particles, we performed MTT and luciferase assays after treating DU145 cells with the SAW and bulk mixed polyplexes of the polymers \#442 and \#442 \& \#689 and the KB cells with the SAW and bulk mixed polyplexes of the polymers \#709 and \#709 \& \#689. Figure 10A shows the obtained MTT values. The DU145 cells show no reduced cell metabolic activity after treatment with SAW and hand mixed 
polyplexes. However, the KB cells do show slightly reduced metabolic activity with no differences between SAW and hand mixed NP.

Figure 10B shows the mean results from a luciferase assay that measures light emitted from firefly luciferase protein when combined with luciferin. Since the pDNA used for polyplex formation codes for the luciferase protein, only transfected cells show bioluminescence. For this experiment KB wildtype and DU145 cells were used. As negative control the cells were treated with HBG-buffer alone, while the positive control consists of well-known bulk-mixed LPEI polyplexes. All used polyplexes show a high transfection efficacy, comparable to or higher than the positive control. In general, polyplexes including the polymer \#689 show a higher transgene expression compared to the polyplexes with polymers \#442 or \#709 alone. One reason for the observed differences could be that the core oligomer \#689 contains a high number of histidines which can improve escape of polyplexes out of endosomes. Additionally, the increased charge to PEG ratio in the three-component formulations provides better pDNA condensation and transfection efficiency as described before [8].

$A$

DU 145 cells KB cells

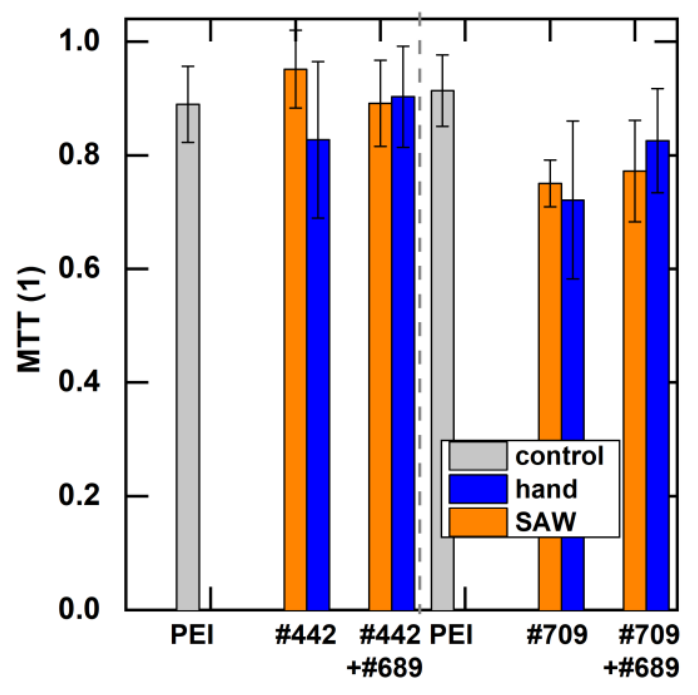

$B$

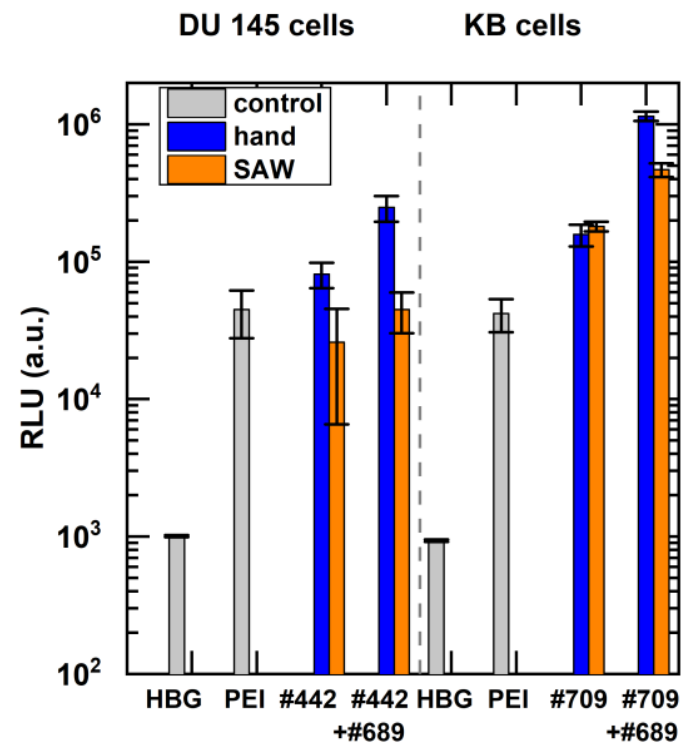

Figure 10: In vitro efficacy A) Metabolic activity of DU145 cells 24 h after treatment with polyplexes of polymers \#442, \#689 and pDNA $\left(c_{p D N A}=2 \mu \mathrm{g} / \mathrm{mL}\right)$ and $K B$ cells $24 \mathrm{~h}$ after treatment with polyplexes of polymers \#709, \#689 and pDNA ( $c_{p D N A}=2 \mu \mathrm{g} / \mathrm{mL}$ ) determined by a MTT assay. B) Gene expression after transcription of DU145 cells $24 \mathrm{~h}$ after treatment with polyplexes of polymers \#442, \#689 and pDNA $\left(c_{p D N A}=2 \mu \mathrm{g} / \mathrm{mL}\right)$ and $K B$ cells $24 \mathrm{~h}$ after treatment with polyplexes of polymers \#709, \#689 and pDNA $\left(c_{P D N A}=2 \mu \mathrm{g} / \mathrm{mL}\right)$ measured by a Luciferase assay.

We attribute the slightly lower in vitro efficacy of the SAW-mixed particles to their smaller size. During in vitro transfections, larger polyplex size can be advantageous due to particle sedimentation under static cell culture conditons[30,35,36]. This artifact can, however, become irrelevant in case of favourable biodistribution of smaller particles under in vivo conditions. 


\section{Conclusion}

Summing up, we have presented an easy to handle and easy to automatize method for formation of nanoparticles. The method is applicable from low to ultralow volumes and comes with the benefits of microfluidic approaches but without the big disadvantage of dead volumes as e.g. in continuous flow systems. We have shown that by tuning concentrations, mixing order and SAW power, we can exert a high degree of control over the size and PDI of polyplexes consisting of pDNA and various cationic polymers. Moreover, we were able to detect small effects like sensitivity towards the mixing order of more-component-polyplexes due to the accuracy of our system. Thus, the technique is ideally suited for a manifold of scientific applications, working with rare or cost-intensive samples but also for screening purposes for pharmaceutical research and development. Beyond the presented applications for therapeutic nanoparticles, SAW based droplet fusion and mixing bears the potential to be a powerful platform for all nanoparticle fabrication processes that are sensitive to the reaction kinetics of particle formation.

\section{Acknowledgments}

The authors would like to acknowledge funding by Nanosystems Initiative Munich (NIM), the Center for NanoScience (CeNS) and the Augsburg Centre for Innovative Technologies (ACIT) for financial support for this project. The authors thank Joachim Rädler for inspiring discussions.

\section{References}

[1] Cho K, Wang X, Nie S, Chen Z and Shin D M 2008 Therapeutic nanoparticles for drug delivery in cancer Clin. Cancer Res. 14 1310-6

[2] Felgner P L, Barenholz Y, Behr J P, Cheng S H, Cullis P R, Huang L, Jessee J, Seymour L W, Szoka F C, Thierry A R, Wagner E and Wu G 1997 Editorial N o m e $n$ c I a t u r e for Synthetic G e n e Delivery Systems Hum. Gene Theraoy 8 511-2

[3] Lächelt U and Wagner E 2015 Nucleic Acid Therapeutics Using Polyplexes: A Journey of 50 Years (and Beyond) Chem. Rev. 115 11043-78

[4] Boussif O, Lezoualc'h F, Zanta M A, Mergny M D, Scherman D, Demeneix B and Behr J P 1995 A versatile vector for gene and oligonucleotide transfer into cells in culture and in vivo: polyethylenimine. Proc. Natl. Acad. Sci. 92 7297-301

[5] Chee G K, Kang X, Xie Y, Fei Z, Guan J, Yu B, Zhang X and Lee L J 2009 Delivery of polyethylenimine/DNA complexes assembled in a microfluidics device Mol. Pharm. 6 1333-42

[6] Lächelt U and Wagner E 2015 Nucleic Acid Therapeutics Using Polyplexes: A Journey of 50 Years (and Beyond) Chem. Rev. 115 11043-78

[7] Kasper J C, Schaffert D, Ogris M, Wagner E and Friess W 2011 The establishment of an up-scaled micro-mixer method allows the standardized and reproducible preparation of well-defined plasmid/LPEI polyplexes Eur. J. Pharm. Biopharm. 77 $182-5$

[8] Kos $P$, Lächelt U, Herrmann A, Mickler F M, Döblinger M, He D, Krhač Levačić A, Morys S, Bräuchle C and Wagner E 2015 Histidine-rich stabilized polyplexes for cMetdirected tumor-targeted gene transfer Nanoscale 7 5350-62 
[9] He D, Müller K, Krhac Levacic A, Kos P, Lächelt U and Wagner E 2016 Combinatorial Optimization of Sequence-Defined Oligo(ethanamino)amides for Folate Receptor-

Targeted pDNA and siRNA Delivery Bioconjug. Chem. 27 647-59

[10] Nguyen N and Wu Z 2004 Micromixers-a review J. Micromechanics Microengineering $15 \mathrm{R} 1-16$

[11] Suh Y K and Kang S 2010 A review on mixing in microfluidics Micromachines 182 111

[12] Lee C Y, Chang C L, Wang Y N and Fu L M 2011 Microfluidic mixing: A review Int. J. Mol. Sci. 12 3263-87

[13] Ma J, Lee S M-Y, Yi C and Li C-W 2017 Controllable synthesis of functional nanoparticles by microfluidic platforms for biomedical applications - a review Lab Chip 17 209-26

[14] Frommelt T, Kostur M, Wenzel-Schäfer M, Talkner P, Hänggi P and Wixforth A 2008 Microfluidic Mixing via Acoustically Driven Chaotic Advection Phys. Rev. Lett. 100 034502

[15] Friend J R, Yeo L Y, Arifin D R and Mechler A 2008 Evaporative self-assembly assisted synthesis of polymeric nanoparticles by surface acoustic wave atomization Nanotechnology 19145301

[16] Westerhausen C, Schnitzler L, Wendel D, Krzysztoń R, Lächelt U, Wagner E, Rädler $\mathrm{J}$ and Wixforth A 2016 Controllable Acoustic Mixing of Fluids in Microchannels for the Fabrication of Therapeutic Nanoparticles Micromachines 7150

[17] Wixforth A 2003 Acoustically driven planar microfluidics Superlattices Microstruct. 33 389-96

[18] Wixforth A, Strobl C, Gauer C, Toegl A, Scriba J and v. Guttenberg Z 2004 Acoustic manipulation of small droplets Anal. Bioanal. Chem. 379 982-91

[19] Ai Y and Marrone B L 2012 Droplet translocation by focused surface acoustic waves Microfluid. Nanofluidics 13 715-22

[20] Schneider C A, Rasband W S and Eliceiri K W 2012 NIH Image to ImageJ: 25 years of image analysis Nat. Methods 9 671-5

[21] Thielicke W and Stamhuis E J 2014 PIVlab - Towards User-friendly, Affordable and Accurate Digital Particle Image Velocimetry in MATLAB J. Open Res. Softw. 2

[22] Stamp M E M, Brugger M S, Wixforth A and Westerhausen C 2016 Acoustotaxis - in vitro stimulation in a wound healing assay employing surface acoustic waves Biomater. Sci. 4 1092-9

[23] Rathgeber A, Strobl C, Kutschera H-J and Wixforth A 2001 Planar microfluidics liquid handling without walls J. Colloid Interface Sci. 357 534-40

[24] K. Dransfeld and Salzman E 1970 Excitation, detection, and attenuation of highfrequency elastic surface waves Physical Acoustics, Principles and Methods, Vol. VII ed W P Mason and R N Thurston (Acad. Press)

[25] Guttenberg Z, Müller H, Habermüller H, Geisbauer A, Pipper J, Felbel J, Kielpinski M, Scriba J and Wixforth A 2005 Planar chip device for PCR and hybridization with surface acoustic wave pump Lab Chip 5 308-17

[26] Tockary T A, Osada K, Motoda Y, Hiki S, Chen Q, Takeda K M, Dirisala A, Osawa S and Kataoka K 2016 Rod-to-Globule Transition of pDNA/PEG-Poly $(<\operatorname{scp}>|</ s c p\rangle-$ Lysine) Polyplex Micelles Induced by a Collapsed Balance Between DNA Rigidity and PEG Crowdedness Small 12 1193-200 
[27] Takeda K M, Osada K, Tockary T A, Dirisala A, Chen Q and Kataoka K 2017 Poly(ethylene glycol) Crowding as Critical Factor To Determine pDNA Packaging Scheme into Polyplex Micelles for Enhanced Gene Expression Biomacromolecules 18 36-43

[28] Intra J and Salem A K 2008 Characterization of the transgene expression generated by branched and linear polyethylenimine-plasmid DNA nanoparticles in vitro and after intraperitoneal injection in vivo J. Control. Release 130 129-38

[29] Yoshikawa K, Minagawa K, Matsuzawa Y and Interactions N 1991 Direct observation of the biphasic conformational induced by cationic polymers $29567-9$

[30] Ogris M, Steinlein P, Kursa M, Mechtler K, Kircheis R and Wagner E 1998 The size of DNA / transferrin-PEl complexes is an important factor for gene expression in cultured cells $1425-33$

[31] Wagner K, Harries D, May S, Kahl V, Rädler J O and Ben-Shaul A 2000 Direct Evidence for Counterion Release upon Cationic Lipid-DNA Condensation Langmuir $16303-6$

[32] Mascotti D P and Lohman T M 1990 Thermodynamic extent of counterion release upon binding oligolysines to single-stranded nucleic acids. Proc. Natl. Acad. Sci. 87 3142-6

[33] DeRouchey J, Netz R R and Rädler J O 2005 Structural investigations of DNApolycation complexes Eur. Phys. J. E 16 17-28

[34] Parker A L, Oupicky D, Dash P R and Seymour L W 2002 Methodologies for Monitoring Nanoparticle Formation by Self-Assembly of DNA with Poly(I-lysine) Anal. Biochem. 302 75-80

[35] Cui J, Faria M, Björnmalm M, Ju Y, Suma T, Gunawan S T, Richardson J J, Heidari H, Bals S, Crampin E J and Caruso F 2016 A Framework to Account for Sedimentation and Diffusion in Particle-Cell Interactions Langmuir 32 12394-402

[36] Wightman L, Kircheis R, Carotta S, Ruzicka R and Kursa M 2001 Different behavior of branched and linear polyethylenimine for gene delivery in vitro and in vivo 362-72 


\section{SUPPORTING INFORMATION}

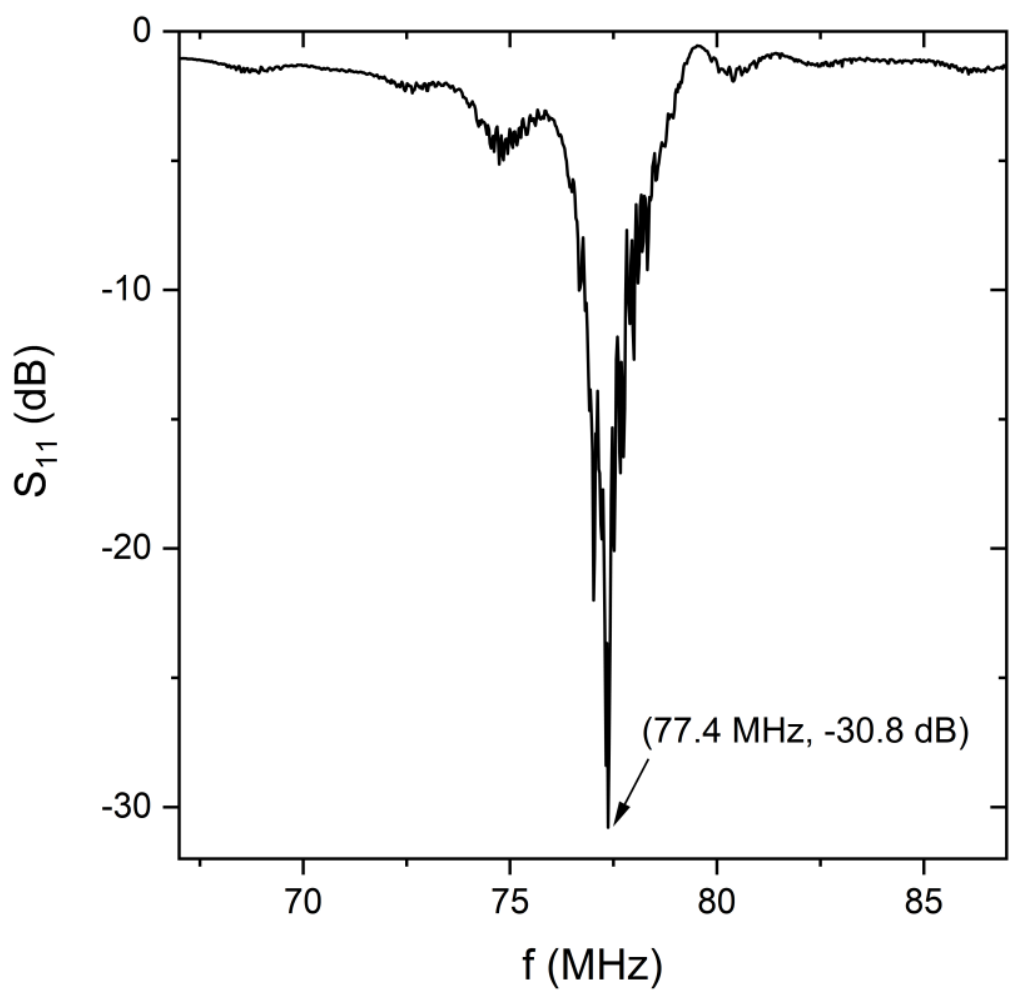

S1: $\boldsymbol{S}_{11}$ measurement The resonance frequency for the in the experiment used IDT is $77.4 \mathrm{MHz}$, measured by a Network Analyzer. Typical values for the reflection coefficient $S_{11}$ are around $-30 \mathrm{~dB}$.
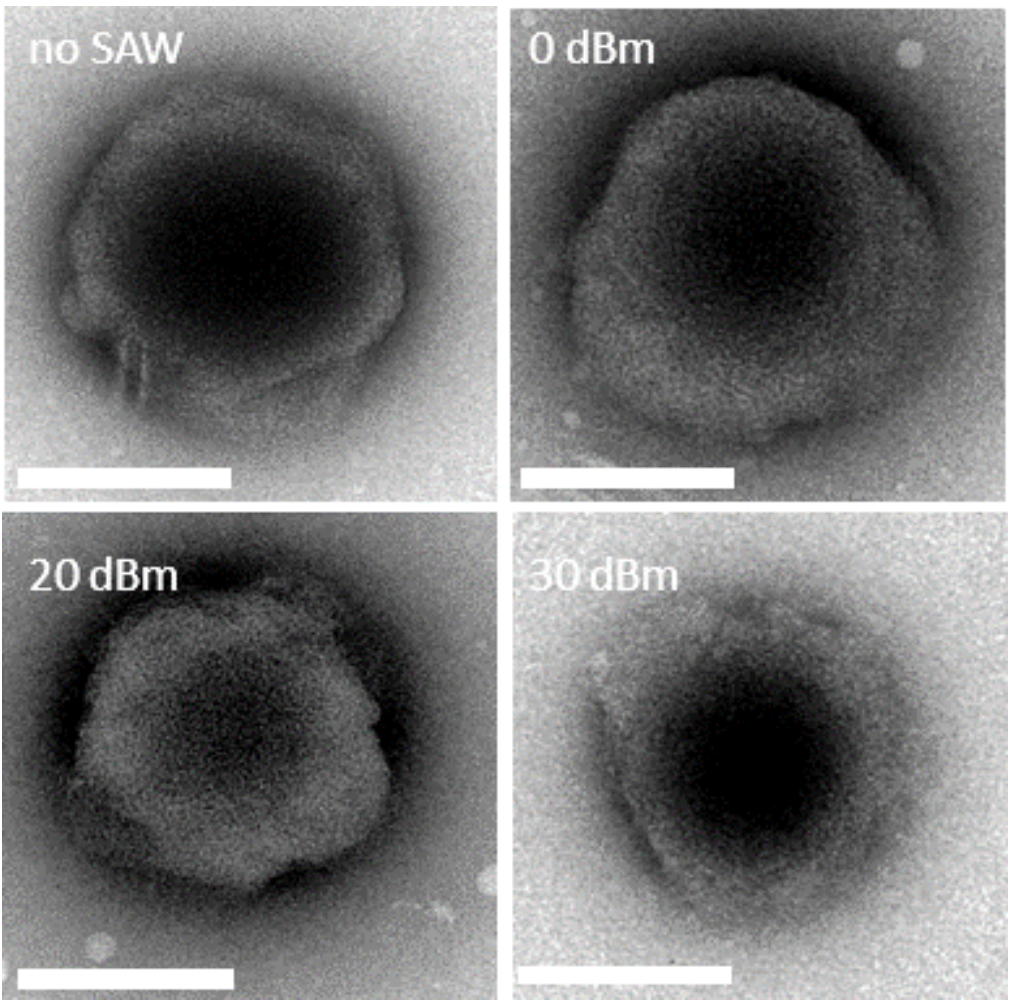

S2: NP morphology Electron migrographs of polyplexes from PEI and pDNA (10 $\mu L P E I+10 \mu L p D N A$, $c_{P E I}=104 \mu \mathrm{g} / \mathrm{mL}, c_{P D N A}=80 \mu \mathrm{g} / \mathrm{mL}$ ) produced by hand mixing and SAW induced mixing using different power levels $P_{\text {SAW }}($ scale bar $=100 \mathrm{~nm}$ ). 